\title{
A 42-year-old woman with subacute reversible dementia: A cautionary tale
}

\section{Joseph Kamtchum-Tatuene ${ }^{1,2}$, Richard Kamwezi ${ }^{3}$, Thokozani Nyalubwe ${ }^{4}$, Josephine P. Banda ${ }^{4}$, Gloria Mwangalika ${ }^{1}$, Ian Matandika ${ }^{3}$, Ulla Hemmila ${ }^{3}$, Magnus Tisell ${ }^{5}$, Susanna Koponen ${ }^{6}$, Laura A. Benjamin ${ }^{2}$, Patrick Kamalo ${ }^{7}$}

1. Malawi-Liverpool-Wellcome Trust Clinical Research Programme, College of Medicine, University of

Malawi, Blantyre, Malawi

2. Liverpool Brain Infections Group, Institute of Infection and Global Health, University of Liverpool,

Liverpool, United Kingdom

3. Department of Internal Medicine, Queen Elizabeth Central Hospital, Blantyre, Malawi

4. Department of Surgery, Queen Elizabeth Central Hospital, Blantyre, Malawi

5. Institute of Neuroscience and Physiology, Sahlgrenska Academy, University of Gothenburg, Gothenburg, Sweden

6. Department of Neurosurgery, NeuroCenter, Kuopio University Hospital, Kuopio, Finland

7. Department of Surgery, College of Medicine, University of Malawi, Blantyre, Malawi

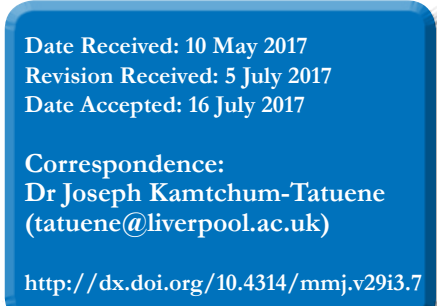

Date Received: 10 May 2017

5 July 2017

ate Accepted: 16 July 2017

ndence:

http://dx.doi.org/10.4314/mmi.v29i3.7

\begin{abstract}
A 42-year-old woman presented with a 6-month history of diffuse headache of moderate intensity and gradual onset of generalized weakness, imbalance, apathy, memory decline, hypophonia, dysphagia, constipation and urinary incontinence. Clinical examination revealed several elements of a frontal lobe dysfunction including apathy with motor impersistence, presence of primitive reflexes, generalized hyperreflexia with bilateral Hoffman sign and ankle clonus. The biological workup was unremarkable and a brain computed tomography scan identified a giant olfactory groove meningioma. A prompt neurosurgical intervention helped to reverse the symptoms. This case illustrates the benefits of actively looking for treatable conditions in young patients presenting with acute or subacute dementia and emphasizes the pivotal role of early brain imaging.
\end{abstract}

\section{Introduction}

Dementia is a syndrome of progressive impairment in one or more areas of cognition. These include memory, language, abstract thinking, praxis, perceptual skills, personality, as well as social behaviour. The cognitive aberrations of dementia usually represent a significant decline from previous baseline capacities, and they interfere with work, social function, relationships and independence in activities of daily living. The prevalence rises from about $1-2 \%$ at age 65 to $10-15 \%$ at age 80 , and may be as high as $40 \%$ by age 90. ${ }^{1}$ Neurodegenerative conditions like Alzheimer's disease and frontotemporal lobar degenerations are, by far, the commonest causes of dementia. ${ }^{2}$ They usually have a poor prognosis given that there is currently no curative treatment for this disorder, and existing symptomatic drugs only have limited effects. ${ }^{3}$ However, there are also several treatable diseases that can lead to dementia with a more or less rapid course. ${ }^{4,5}$ This case report aims to emphasize the need to actively look for treatable causes in patients presenting with dementia, especially when there are some atypical clinical features.

\section{Case presentation}

A 42-year-old woman presented in the emergency department with a 6-month history of diffuse headache of moderate intensity. Over a course of 3 months, she also had gradual onset of imbalance, generalized weakness, hypophonia, apathy, dysphagia, constipation and urinary incontinence. The guardians reported a change in her personality over the previous years with frequent episodes of incoherent speech and memory decline. Her maternal aunt had early-onset cognitive decline and died before the age of 50 . There was no history of head trauma or fluid leakage through the ear, the nose or the throat. The following symptoms were absent: seizures, hallucinations, vomiting, diplopia, blurred vision, agitation, and sensory loss.
On admission, she was afebrile and bedridden. The neurological examination identified a severe apathy with motor impersistence, marked primitive reflexes (sucking, palmomental, and Babinski sign), generalized hyperreflexia with bilateral Hoffman sign and ankle clonus but no limb spasticity, an effortful but comprehensible speech, and frequent startles. There was no papillary oedema on the fundoscopy. Because of the severe apathy and somnolence, it was not possible to perform a more precise evaluation of the olfaction, the higher order cerebral functions, and the motor and sensory pathways.

Given the limited resource available, the patient underwent a minimal biological workup. The full blood count, the liver and thyroid function tests and the HIV and syphilis screening tests were unremarkable. The cerebrospinal fluid analysis revealed a high protein level $(1.9 \mathrm{~g} / \mathrm{L})$ with normal cell count, microscopy and culture. The kidney function could not be assessed for technical reasons. A brain computed tomography (CT) scan without contrast (unknown creatinine level) revealed a large irregular paramedian subfrontal extraaxial mass, slightly hyperdense with some calcifications and a local mass effect, with effacement of frontal sulci (Figure 1A).

The radiological features were suggestive of an olfactory groove meningioma. The patient had a neurosurgical resection of the tumour (shown in Figure 1B) leading to a significant improvement of her symptoms. The histological examination confirmed a grade I meningothelial meningioma. There was no immediate post-operative complication. The post-operative MRI confirmed the complete resection of the tumour and the re-expansion of the frontal lobes (Figures $1 \mathrm{C}$ and 1D). The post-operative follow-up was uneventful and the patient was asymptomatic and independent 6 weeks after the surgery. 


\section{Discussion}

This patient presented with a dementia (progressive impairment of memory, social behaviour and personality with loss of independence) associated with signs of frontal lobe dysfunction (apathy, hypophonia, dysphagia, urinary incontinence, motor impersistence, marked primitive reflexes and generalized hyperreflexia). In the context of a positive family history for early-onset dementia, the diagnosis of fronto-temporal dementia (FTD) could be proposed. The latter is considered as the second most common cause of early onset dementia (before the age of 65), second only to Alzheimer's disease. ${ }^{6}$ Approximately $40 \%$ of FTD is associated with an autosomal dominant pattern of inheritance. ${ }^{7}$ However, given the young age, the presence of headache and the rapid progression leading to severe disability in less than a year, other non-degenerative and potentially treatable conditions needed to be discussed. ${ }^{5}$ These conditions include brain tumours involving the frontal lobe (especially parafalcine or olfactory groove meningiomas with symmetrical involvement of both frontal lobes and compression of the micturition control centre in the medial frontal cortex), auto-immune encephalitis, hydrocephalus (obstructive or normal pressure; the latter typically causes gait instability, urinary incontinence and dementia [HakimAdams' triad]), infectious diseases (HIV-associated neurocognitive decline, neurosyphilis, sporadic CreutzfeldtJakob disease), chronic post-traumatic lesions (subdural hematoma), metabolic diseases (e.g. hypothyroidism), and depression. Reversible dementia has also been reported with various drugs, notably anticholinergics, benzodiazepines, antidepressants, steroids, opiates, non-steroidal antiinflammatory drugs and histamine $\mathrm{H} 2$ receptor antagonists. ${ }^{8}$ In this case, a well selected set of paraclinical examinations helped to quickly identify a giant olfactory groove meningioma as the cause of the dementia and to provide the appropriate treatment in order to reverse the symptoms. Meningiomas are the most common meningeal tumours and represent more than $20 \%$ of all intracranial tumours. ${ }^{9}$ They usually affect women in their fifth decade. Neurofibromatosis type $2,{ }^{10}$ irradiation, ${ }^{11}$ hormone replacement therapy, ${ }^{12}$ and foreign bodies ${ }^{13}$ are classical risk factors but were not present in our case. Head trauma has also been suspected as a risk factor for meningiomas in case-reports and casecontrol studies but the association is not confirmed in population-based cohorts. ${ }^{14,15}$ This suggests the influence of a recall bias in case-control studies. Meningiomas can be found in all locations but $60 \%$ of meningiomas are either parasagittal (inserted on the falx) or located on the convexity. The olfactory groove location is observed in less than $10 \%$ of cases. ${ }^{16}$ When meningiomas are slow growing and located in the anterior frontal region as is the case in our patient, the diagnosis is frequently delayed. This happens because focal neurological deficits are often absent and the intracranial pressure is only slightly elevated if at all. Moreover, in some patients, a chronic papilledema might be difficult to identify on fundoscopy due to secondary optic nerve atrophy.

Olfactory groove meningiomas frequently present as pseudodementia resulting from subacute or chronic compression of the frontal lobe. ${ }^{16}$ This chronic compression of the central nervous system might induce a high protein level in the cerebrospinal fluid, a non-specific finding frequently reported with spinal cord compressions. The sensitivity

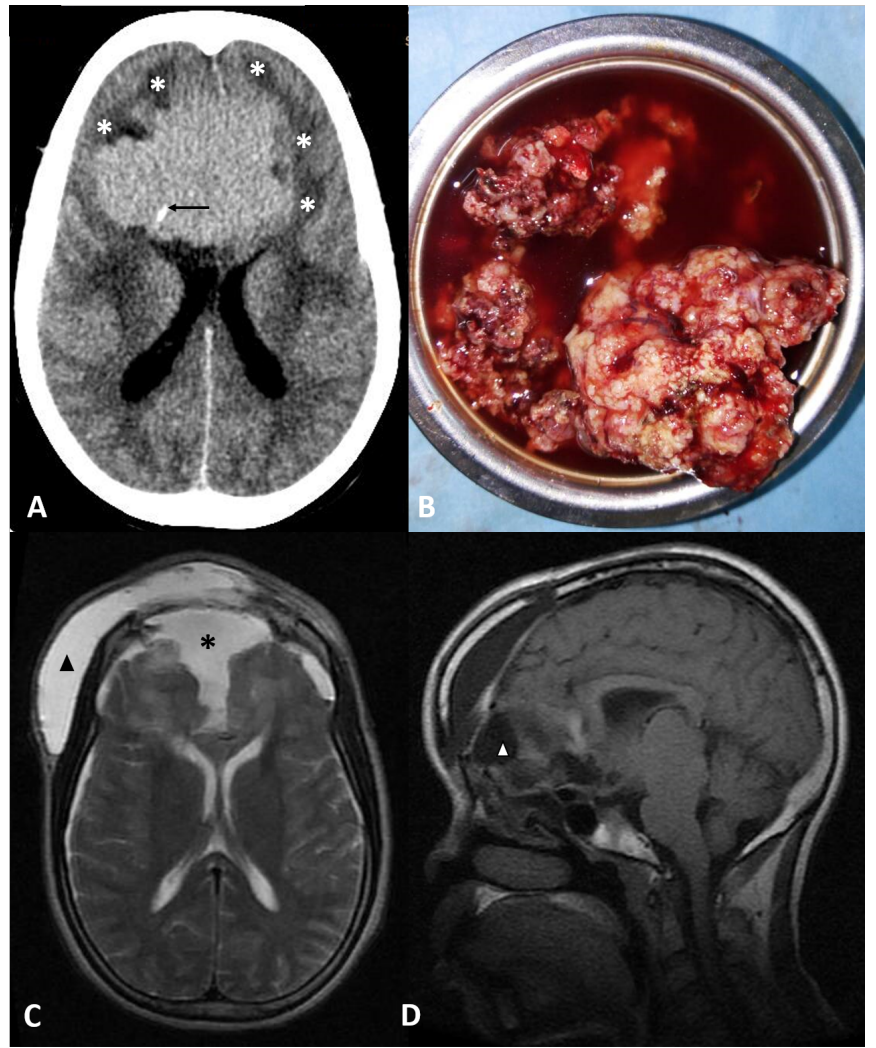

Figure 1: Olfactory groove meningioma - pre- and post-operative images

A: Preoperative axial brain computed tomography (CT) scan slice (without contrast) showing the olfactory groove meningioma as an extra-axial hyperdense mass with a small calcification (black arrow). There is significant perilesional oedema in the frontal lobes (white asterisks) and evidence of mass effects on the anterior horns of the lateral ventricles. B: Macroscopic view of the meningioma freshly excised. The bowl has the shape of a truncated cone (outlet inner diameter: $16 \mathrm{~cm}$, base diameter: $11 \mathrm{~cm}$, depth: $6 \mathrm{~cm}$ ). C: Post-operative axial brain T2 magnetic resonance imaging (MRI) slice showing the complete resection of the olfactory groove meningioma (black asterisk). The lateral ventricles have recovered their normal shape. The frontal bone flap was not tightly fixed back for safety reasons. This explains the presence of a subcutaneous cerebrospinal fluid pouch (black arrow head). D: Post-operative sagittal brain T1 MRI slice also showing the complete resection of the olfactory groove meningioma (white arrow head) and the normal re-expanded frontal lobe.

of a brain CT scan for the diagnosis is almost $100 \%$. Meningiomas typically appear as hyperdense or isodense extra-axial lesions, inserted on the meninges, with inconstant calcifications and homogeneous contrast enhancement. Associated bone lesions are frequently found, notably hyperostosis or osteolysis. ${ }^{9,17}$ Meningiomas are most often benign and surgical treatment is usually associated with very good prognosis and very low recurrence rate.

This case illustrates the benefits of actively looking for treatable conditions in young patients presenting with acute or subacute dementia. It also emphasizes the role of early brain imaging. In resource-limited settings where careful selection of complementary examinations is mandatory, investigation of dementia should always include brain imaging as soon as possible, unless there are other obvious infectious, hemodynamic or metabolic emergencies.

\section{Competing interests}

All authors declare that they have no competing interests related to this work.

\section{Acknowledgements}

The authors are grateful to the patient and her relatives for granting the authorization to use their medical information for teaching and scientific publication. 


\section{References}

1. Galasko D. The diagnostic evaluation of a patient with dementia. Continuum (Minneap Minn) 2013;19(2 Dementia):397-410.

2. Kelley BJ, Boeve BF, Josephs KA. Young-onset dementia: demographic and etiologic characteristics of 235 patients. Arch Neurol 2008;65(11):1502-1508.

3. Sorbi S, Hort J, Erkinjuntti T, et al. EFNS-ENS Guidelines on the diagnosis and management of disorders associated with dementia. Eur $\mathrm{J}$ Neurol 2012;19(9):1159-1179.

4. Masdeu JC, Pascual B. Neuroimaging of disorders leading to dementia. Curr Neurol Neurosci Rep 2008;8(6):443-444.

5. Murray K. Creutzfeldt-Jacob disease mimics, or how to sort out the subacute encephalopathy patient. Pract Neurol 2011;11(1):19-28.

6. Finger EC. Frontotemporal Dementias. Continuum (Minneap Minn) 2016;22(2 Dementia):464-489.

7. Rohrer JD, Guerreiro R, Vandrovcova J, et al. The heritability and genetics of frontotemporal lobar degeneration. Neurology 2009;73(18):1451-1456.

8. Gray SL, Lai KV, Larson EB. Drug-induced cognition disorders in the elderly: incidence, prevention and management. Drug Saf 1999;21(2):101-122.

9. Dietemann JL, Eid MA, Soares IM, Bogorin A, Boyer P, Draghici S. Tumeurs cranioencephaliques: tumeurs extra-axiales. In: Dietemann JL, editor. Neuro-imagerie diagnostique. 2nd ed. Paris: Elsevier Masson; 2012. p. 305-373.
10. Goutagny S, Kalamarides M. Meningiomas and neurofibromatosis. J Neurooncol 2010;99(3):341-347.

11. Yamanaka R, Hayano A, Kanayama T. Radiation-Induced Meningiomas: An Exhaustive Review of the Literature. World Neurosurg 2017;97:635-644 e638.

12. Fan ZX, Shen J, Wu YY, Yu H, Zhu Y, Zhan RY. Hormone replacement therapy and risk of meningioma in women: a metaanalysis. Cancer Causes Control 2013;24(8):1517-1525.

13. Saleh J, Silberstein HJ, Salner AL, Uphoff DF. Meningioma: the role of a foreign body and irradiation in tumor formation. Neurosurgery 1991;29(1):113-118; discussion 118-119.

14. Kuan AS, Chen YT, Teng CJ, Wang SJ, Chen MT. Risk of meningioma in patients with head injury: a nationwide populationbased study. J Chin Med Assoc 2014;77(9):457-462.

15. Nygren C, Adami J, Ye W, et al. Primary brain tumors following traumatic brain injury--a population-based cohort study in Sweden. Cancer Causes Control 2001;12(8):733-737.

16. Hentschel SJ, DeMonte F. Olfactory groove meningiomas. Neurosurg Focus 2003;14(6):e4.

17. Lee KF. The diagnostic value of hyperostosis in midline subfrontal meningioma. Radiology 1976;119(1):121-130. 